\section{Gaston Gross}

Université Paris 13

France

(D) https://orcid.org/0000-0003-2786-4178
Des perspectives rigoureuses pour la linguistique

\title{
Rigorous perspectives on linguistics
}

\begin{abstract}
A reading of many recent publications shows that theoretical concerns in the field of linguistics have declined, even though the founders of the discipline all emphasised the central importance of its role. This article aims to show how linguistic description has progressed thanks to the different theoretical tools that have been developed in the course of research. From this point of view, this article illustrates the following facts: lexicon, semantics and syntax are not separate instances but together form the units that are sentences. The elements of sentences must be described in terms of the set of properties that characterise them. This is the case for regular sequences. Finally, one of the promising objectives consists in finding a reasoned classification of fixed sequences. Linguistics, like any science, cannot do without theoretical tools.
\end{abstract}

\section{Keywords}

Theories, theoretical requirement, scientific rigour, research opportunities, usefulness

\section{Introduction}

Il est assez habituel de constater chez les linguistes, formés dans les années 80 , l'opinion que la linguistique a perdu ces dernières années de sa rigueur théorique. Face à ce constat, différentes réactions sont possibles. On peut penser que l'exigence théorique a été, à un moment donné, négligée à la fois dans l'examen des thèses ou lors des recrutements, d'autant que l'histoire de la linguistique a toujours été associée à des considérations exigeantes du point de vue théorique. Les fondateurs de la discipline ont adopté des positions claires et déterminées sur le rôle primordial de l'outil théorique. Tous les travaux marquants des cinquante 
dernières années ont mis ces préoccupations au premier plan. Il suffit de citer, parmi d'autres, les noms de : A. Meillet, F. de Saussure, Z. Harris, N. Chomsky, M. Gross, J. Dubois. Au lieu de se complaire en regrets, on peut aussi proposer des perspectives susceptibles de faire progresser notre discipline. Je voudrais montrer comment la recherche peut évoluer aux différents stades des travaux. J'examinerai, dans un premier temps, les dictionnaires classiques, je résumerai ensuite les recherches réalisées par et sous la direction de Maurice Gross au LADL, je proposerai enfin des perspectives de recherches qui allient une exigence théorique et une utilité en vue du traitement automatique.

\section{Les dictionnaires-papier classiques}

Les dictionnaires-papier du commerce reposent sur un certain nombre de principes méthodologiques, que l'on pourrait résumer de la façon suivante :

- les entrées sont constitués de mots mono-lexicaux ;

— un dictionnaire a pour objectif principal la description des mots dits « pleins »;

— on constate des niveaux d'analyse différents : le lexique (dictionnaire), la syntaxe (grammaire), la sémantique (qui n'a pas d'ouvrages spécifiques, sauf des études théoriques) ;

- le cadre théorique est celui des catégories grammaticales, dont on ne remet pas en cause les définitions classiques. En particulier, les dictionnaires ne connaissent pas la notion de prédicat nominal ni de verbes supports ;

- la détermination des arguments et la conjugaison des prédicats, en particulier des prédicats nominaux, ne trouvent pas leur place dans un dictionnaire ;

- la notion d'emploi de prédicat est inconnue.

Voici le traitement du substantif regard dans les dictionnaires les plus connus. La description est particulièrement ténue.

a) Le Petit Larousse illustré (PLI) 1991

1. action, manière de regarder. Attirer tous les regards

2. Expression des yeux : un regard tendre

b) LEXIS (Larousse, 1975) s.v. regarder

1. Action de regarder jeter, lancer un regard sur quelqu'un (syn. Coup d'œil) parcourir du regard (examiner), menacer $d u$ regard, soustraire au regard (syn:vue)

2. Manière de regarder, expression des yeux : un regard doux, tendre, caressant, languissant ; Un regard sombre, terrible, menaçant, méprisant, hautain ; un regard vif, pénétrant, perçant 
3. Tourner ses regards vers : implorer, mettre son espoir dans

c) Le Petit Robert (1993)

1. Action, manière de diriger les yeux vers un objet, afin de voir ; expression des yeux de la personne qui regarde. Le regard humain. Parcourir, explorer, fouiller, suivre du regard (regarder), dérober, soustraire aux regards (cacher). Dévorer du regard, menacer, foudroyer du regard

L'expression habituelle des yeux : regard candide, malicieux, expressif, perçant

Un regard : un coup d'œil : un regard rapide, distrait, furtif; au premier regard : au premier coup d'œil. Des regards en coin, en coulisse, un regard étonné

2. Action de considérer avec attention : avoir droit de regard sur

Comme on le voit, les informations sont plus précises dans les deux derniers, mais des informations essentielles sont omises : les verbes supports et les verbes appropriés n'y sont pas discriminés, d'où l'ignorance de toute une partie théorique des prédicats nominaux.

\section{Les outils théoriques du LADL}

Les travaux du LADL et leur apport dans la description linguistique sont bien connus. Je résumerai ici les perspectives théoriques de cette équipe de recherche. a) L'unité minimale d'analyse n'est pas le mot mais la phrase. Toute entrée de dictionnaire est donc constituée d'un prédicat et de la suite la plus longue de ses arguments.

b) La première étape de la recherche consiste à dresser la liste des phrases élémentaires. Les propriétés de distribution, c'est-à-dire celles qui décrivent la nature des arguments, sont précisées au fil des recherches par des informations de nature morphologique : soit un substantif $(N)$ munis d'un certain nombre d'indices (Nhum, Nc, Nloc) soit une phrase $\mathrm{P}$ (en fait une complétive de forme le fait que $P$, que $P$, ou la réduction infinitive $V W$ ).

c) Les transformations, elles, impliquent des changements morphologiques du prédicat (verbe, nom, adjectif) ou de structure de la phrase (passif, réduction infinitive, pronominalisation, interrogation). Les propriétés, codées en abscisse de façon binaire $(+,-)$, permettent de déterminer les différentes formes que peut prendre une phrase définie par les particularités d'un prédicat donné. Chaque ligne représente donc les informations nécessaires à la reconnaissance d'un emploi. 


\subsection{Codage des arguments dans les tables}

Nous venons de dire que les arguments d'un prédicat peuvent être des noms ou des verbes. Il n'a jamais été question de préciser, dans les tables, la nature sémantique des verbes figurant dans les structures : le fait que $P$, que $P$ ou dans la réduction infinitive : le fait de $V W$. Et pourtant, elle a la même importance que celle des substantifs qui se trouvent dans les positions argumentales parallèles. Cette absence s'explique par le fait qu'il n'existait à l'époque aucune classification des prédicats : tout complément phrastique est uniformément noté par les suites catégorielles qui viennent d'être citées, indépendamment du sens de ces verbes. Il est clair pourtant qu'un prédicat comme entreprendre est suivi d'un verbe d'action (entreprendre de coder ces mots) et non pas d'un verbe événementiel comme pleuvoir, par exemple.

Mais le problème se pose plus crucialement quand les arguments, au lieu d'être des verbes, sont de nature nominale. La réticence de la grammaire distributionnelle à recourir à la sémantique apparaît clairement dans la façon dont M. Gross sous-catégorise les noms. À l'évidence, il n'est pas suffisant de coder les arguments nominaux en se contentant de les noter par $N$, sinon les verbes auraient tous le même type de sujets ou de compléments. M. Gross n'avait pas non plus dans son outillage théorique une classification sémantique des substantifs, à laquelle il se refusait au surplus. Malgré cette réticence, il n'abandonne pas entièrement la partie. "Nous pensons néanmoins qu'il est possible d'effectuer diverses opérations sur le sens dans de bonnes conditions expérimentales » (1975 : 30). Il est possible, dit-il, grâce à la syntaxe d'établir des distinctions sémantiques relativement fines. Ainsi, le verbe amuser peut avoir une double lecture active et non-active. Ces deux lectures sont mises en évidence par la possibilité, pour la lecture non active, d'être complétée par un complément de nature circonstancielle : Pierre amuse Paul par sa façon originale de s'habiller. L'action de Pierre sur Paul est involontaire et sans doute aussi inconsciente. Cependant ces différences sémantiques très fines ne constituent pas des outils permettant de sous-catégoriser les substantifs en position argumentale, même s'il est possible d'attribuer, comme le dit $\mathrm{M}$. Gross, le trait humain à des locatifs grâce à des verbes à objet direct humain : L'orateur a ennuyé la salle. De même, des collectifs abstraits comme administration sont interprétés comme ayant le trait humain grâce au même verbe : Ces habitudes ennuient l'administration. M. Gross note cependant que ces extensions ont des propriétés différentes : Il y a chez Paul une volonté de nuire; *Il y a chez l'administration une volonté de nuire. On retiendra que ces observations sont loin d'être suffisantes pour déterminer la nature argumentale des milliers de verbes du français. 


\subsection{Elimination de l'ambiguïté à l'aide de ces outils}

Une telle description, malgré son caractère schématique, permet de séparer un certain nombre d'emplois de verbes prédicatifs. C'est le cas, par exemple, des verbes abattre et compter.

abattre

Table 4 : Que P V N1hum : Que Paul soit mort a abattu Luc

Table $32 \mathrm{H}: \mathrm{N} 0 \mathrm{~V}$ N1 hum : Le soldat a abattu le prisonnier

compter

5 : Que P V Prép N1hum : Que Max ait dit cela compte pour Luc

6: NOhum V Que P : Luc compte qu'il fera beau

35R : N0hum V Prép N1 : Max comptera avec la pluie

Mais il est clair que cette description, qui se fonde sur le trait générique humain, ne permet pas de générer des phrases correctes à tous les coups. L'emploi de abattre de la table $32 \mathrm{H}$ ne précise pas la suite la plus longue, car ce verbe a un complément indirect mettant en jeu une arme à feu : Le soldat a abattu le prisonnier au pistolet automatique. De même, dans la description des tables 5 et 35R, il ne suffit pas d'indiquer que le complément du verbe compter est indirect, il faut préciser la nature des prépositions en jeu, respectivement pour et avec. De façon plus générale, ces outils ne permettent pas de séparer tous les emplois de ces deux verbes, c'est-à-dire de discriminer la nature sémantique de leurs arguments.

\subsection{Insuffisance des tables}

Cette description, qui se fonde sur des traits syntaxiques génériques (Nhum, N-hum, Nconcret, Nloc), ne peut pas rendre compte de la totalité de la distribution des verbes. Ainsi, la Tablel de Méthodes en syntaxe (1975) est définie de la façon suivante :

\section{NO V Vinf $W$}

De deux choses l'une : ou bien il existe entre les 75 verbes de cette table une homogénéité syntaxique et sémantique telle que le regroupement est justifié ou bien alors, il n'y avait aucune raison de mettre au point cette table. Je critique l'idée que la syntaxe soit première, qu'elle constitue un moule dans lequel on déverse de la sémantique. En effet, cette table génère des constructions totalement hétérogènes. On y trouve ainsi en position verbale : 
— de vrais verbes aspectuels : achever de, aller, arrêter de, cesser de, commencer à, commencer de, commencer par, continuer à, continuer de, finir de, finir par, recommencer à ;

— des verbes d'activité : se dépêcher de, se grouiller de, se hâter de, se magner de, se presser de ;

- des verbes psychologiques ou intellectuels : choisir entre, hésiter à, se contenter de, daigner, se déballonner de, se décider à, se défiler de, se dégonfler de, se dégrouiller de, se démerder de;

- des verbes d'opérations de l'esprit : omettre de, oublier de, opter pour, pencher pour ;

— des verbes modaux : devoir, se devoir de, pouvoir ;

- des verbes d'efforts : s'efforcer de, s'empresser de, tenter de.

On est obligé de conclure que $\boldsymbol{V} \boldsymbol{V}$ inf $\boldsymbol{W}$ est une suite hétérogène qui ne permet pas de décrire une structure déterminée. On doit alors admettre qu'une phrase ne peut pas être définie par un socle constitué d'une séquence abstraite de catégories mais par un prédicat réel, c'est-à-dire un ou des mots lexicaux ouvrant des positions argumentales définies sémantiquement, correspondant à des classes de substantifs. La position théorique qui consiste à dire que la syntaxe, du fait de son caractère falsifiable, est le fondement de l'analyse linguistique est une fausse bonne idée. Au lieu de postuler une hiérarchie entre les différents modules, il faut les intégrer, en prenant en compte le fait qu'il n'existe d'autonomie réelle pour aucun d'entre eux.

\section{Prise en compte de la sémantique}

\subsection{La notion de classes d'objets}

Le trait syntaxique Nhum permet, certes, de discriminer des emplois de prédicats qui ont un sujet différent :

\section{Paul est tombé}

Le résultat est tombé

L'arbre est tombé

Mais avec des verbes plus spécialisés, cette indication est insuffisante. Les classes d'objets d'humains constituent des indications beaucoup plus précises. Voici un certain nombre de classes d'humains, qui offrent une description précise de la relation entre un prédicat donné et ses arguments : 
adepte : protestant, taoïste

ape (appellatif) : monsieur, sire

col : foule, troupe

déf (défauts) : menteur, voleur, imbécile, traître

doct (doctrine) : catholicisme, jansénisme

écr (écrit) : livre, Bible

fon (fonction) : adjoint, arbitre

grade : capitaine, colonel

ins (instrumentiste) : violoniste, pianiste

loc (locatif) : Parisien, Bitterrois, Allemand

locm (locatif par métonymie) : rue, salle

ltr (lieu de travail) : usine, bureau

mal (maladies) : tuberculeux, cancéreux

npra (nom prédicatif actif) : fumeur, conducteur

nprd (nom prédicatif datif) : bénéficiaire

nprp (nom prédicatif passif) : pensionné, élu

pro (profession) : menuisier, chauffeur

qua (qualités) : pieux, vertueux, intelligent

rel, col (relationnel collectif) : famille

rel (relationnel) : frère, père

sportif : footballeur, escrimeur

\subsection{Application de la notion de classes d'objets}

Prenons l'exemple du verbe abattre, qui a au moins 8 emplois différents. Ces significations ne peuvent être mises en évidence par l'indication du seul sujet : Paul abat**. Si l'on indique, en revanche, la nature sémantique de l'objet, alors les différentes significations apparaissent clairement :
Abattre/NO : hum $/ N$ : arbre
Abattre $/ \mathrm{NO}:$ hum $/ \mathrm{N}:$ aéronef
Abattre/NO : hum/N : construction
Abattre/NO : hum/N1 : hum
Abattre/NO : évé/N : hum
Abattre/NO : hum $/ N$ : cartes
Abattre $/ N O:$ hum $/ N:$ minerai
Abattre/NO : hum/N : animal de boucherie

Ces classes sémantiques permettent de mettre en évidence les synonymes de chaque emploi de ce verbe : 
Abattre/NO : hum/N : arbre : couper

Abattre $/ N 0:$ hum $/ N$ : aéronef : descendre

Abattre/NO : hum $/ N$ : construction : démolir

Abattre/NO : hum/N1 : hum : tuer avec une arme à feu

Abattre/NO : évé/N : hum : démoraliser

Abattre/NO : hum/N : cartes : déposer

Abattre/NO : hum $/ N$ : minerai : extraire, détacher

Abattre/NO : hum $/ N$ : animal de boucherie : tuer

ainsi que sa traduction :

Abattre/NO : hum/N : arbre/Sy : couper/E: to cut down/D: fällen Abattre/NO : hum/N : avion/Sy : descendre/E: to shoot down/D: abschiessen Abattre/NO : hum/N : cloison/Sy : démolir/E: to pull down/D: niederreissen Abattre/No : hum/N1 : hum/Sy : exécuter/E: to shoot down/D: erschiessen Abattre/NO : évé/N : hum/Sy : démoraliser/E: to demoralize/D: niederdrücken Abattre/NO : hum $/ N$ : cartes/Sy : déposer/E: to lay down/D: aufdecken Abattre/NO : hum/N : minerai/Sy : détacher/E: to break away/D: ausbrechen Abattre/NO: hum/N: animal de boucherie/Sy: tuer/E: to slaughter/D: schlachten

La notion de trait syntaxique au sens du LADL n'apporte donc pas les informations nécessaires à la reconnaissance des différents emplois, comme on vient de le voir.

\section{Seconde étape de la description : le substantif regard}

Compte tenu de cette modification de l'outil théorique, menant à une description appropriée au traitement automatique, on peut proposer une description plus appropriée des prédicats. Soit le substantif prédicatif regard. La nature sémantique des arguments permet de discriminer ses différents emplois.

\subsection{Emploi intransitif : regard comme prédicat de propriété}

Il existe un emploi sans complément, mais avec un modifieur obligatoire :

*Paul a un regard

Paul a un regard terne 
Cet emploi est défini par :

— un argument-sujet humain (éventuellement animal), il n'y a pas de complément ;

- le verbe support est avoir ;

— on trouve aussi : posséder ;

— un modifieur décrivant le regard est obligatoire : ardent, étincelant, brûlant, terne, vide, fuyant, mobile, vitreux mais non en coin, en biais ;

- le déterminant de regard est l'indéfini un ou le défini le au singulier : Paul a (le, un) regard vif. Le pluriel est interdit *Paul a les regards vifs;

— regard est un prédicat de propriété plutôt que d'action : Son regard est (vif, terne) ;

- l'interprétation est passagère ou habituelle : Paul a un regard terne (ce matin, habituellement) ;

— le substantif regard peut être remplacé par le substantif æil : Paul a l'œil (vif, terne). Le pluriel est moins bon : Paul a les yeux vifs, l'indéfini serait meilleur Paul a des yeux vifs;

— le support peut être effacé : Le regard (vif, terne) de Paul ;

— il n'y a pas de montée de l'adjectif : *Paul est (terne, vif) (de, du) regard;

- la construction impersonnelle est possible : Il y a (de la vivacité, de la mobilité, du feu) dans le regard de Paul ;

- cet emploi n'est pas associé au verbe regarder.

Le substantif regard désigne une propriété de Paul. Elle est moins physique que : Paul a les jambes arquées, peut-être du fait que les yeux peuvent traduire des sentiments, un état d'esprit, certaines facultés, comme la présence d'esprit. Au XVII ${ }^{e}$ siècle on appelait les yeux, le "miroir de l'âme ». Regard n'a rien à voir ici avec le substantif vue: Paul a une vue perçante.

\subsection{Emploi transitif avec modifieur : prédicat de comportement}

Paul a eu un regard (amical, dédaigneux) pour Jean

— Le sujet est humain ; le complément est humain aussi ou désigne une activité humaine introduite par pour ;

- Deux autres supports sont possibles : accorder, concéder, la préposition est alors à : Paul lui a accordé un regard attentif. Le verbe jeter traduirait plutôt une activité qu'un comportement ;

- Le déterminant est indéfini Paul a eu un regard amical pour Jean; le pluriel n'est pas très clair : Paul a eu des regards amicaux pour Jean. Le défini est impossible: *Paul a eu le regard (amical) pour Jean; le possessif est impossible aussi : *Paul a eu son regard pour Jean; 
— Un modifieur est obligatoire, sauf à la forme négative : Paul n'a même pas eu un regard pour Jean. Mais il s'agit peut-être d'une suite figée ;

- Le support est effaçable, mais il faudrait examiner un grand corpus : Le regard dédaigneux de Paul pour Jean ; Son regard dédaigneux pour Jean ;

- Regard peut difficilement être remplacé par cil : ?L'œil dédaigneux de Paul pour Jean ;

- L'adjectif est de nature comportementale : amical, attentif, dédaigneux, hautain mais non descriptif : fixe, fuyant, mobile, acéré, vif, perçant ;

- Il existe une construction impersonnelle : Il y a eu un regard dédaigneux pour Jean de la part de Paul ;

- On constate une autre thématisation : J'ai eu droit à un regard amical de sa part;

- On trouve aussi une construction verbale associée : Paul a regardé Jean dédaigneusement. Dans ce cas, regarder n'est pas un vrai prédicat de perception.

\subsection{Emploi de perception active}

Paul a jeté un regard furtif sur le tableau

Paul a jeté un regard rapide dans cette direction

- Le sujet est humain et l'objet désigne un concret ou un locatif ;

- Les supports sont métaphoriques et issus de causatifs de mouvement fondés sur l'idée de lancer : balancer, lancer, envoyer, filer ;

- Les prépositions spatiales précisent qu'il s'agit de compléments locatifs : vers, en direction de. Si le complément est concret alors la préposition est essentiellement sur. S'il s'agit d'une activité, la préposition peut être à : Je vais jeter un regard à ton texte. Si le complément est humain, à et sur peuvent induire des interprétations différentes : jeter un regard sur (surveiller); jeter un regard à (entrée en communication) ;

- Les déterminants peuvent être : un, des suivis de modifieurs; sont interdits le ou peut-être le possessif : ?Paul a jeté son regard sur ce texte ;

- Il existe des substantifs synonymes : eil : jeter un cil (à, sur) $N$; jeter les yeux (*à, vers, sur) $N$; jeter un coup d'œil (à, sur) $N$;

- Il s'agit d'un prédicat de perception active ;

- Les adjectifs sont moins restreints : en biais, en coin, en coulisse, oblique, furtif ;

— On observe la réduction du verbe support : Le regard de Paul à Jean ; ?Le regard de Paul sur ce texte;

- L'interprétation est celle d'une action rapide : Jeter un regard rapide sur ce texte;

- On observe aussi un verbe associé : Paul m’a regardé en coin. 
La nature du support et la métaphore qui l'explique impliquent une action volontaire et consciente. Il peut y avoir contamination avec l'emploi précédent : Il a eu un regard amical pour Jean; Il a jeté un regard amical à Jean.

\subsection{Emploi de perception active, métaphore fondée sur les armes de jet}

\section{Paul décoche un regard agressif à Jean}

- Le sujet est humain, de même que l'objet ;

- D'autres supports sont possibles : darder ; la préposition est alors plutôt sur que $\grave{a}$. Les dictionnaires définissent ainsi le verbe décocher : lancer avec un arc, une arme de trait; darder: lancer (une arme, un objet) comme on ferait d'un dard. On peut expliquer ainsi la métaphore : assassiner du regard;

- Les déterminants peuvent être un suivi d'un modifieur (ou une intonation exclamative) ; le pluriel est possible : Il lui décocha des regards assassins ; on ne trouve ni le défini et ni le possessif : *Il lui décocha son regard;

- Aspectuellement, il s'agit d'une action rapide : *décocher un long regard de désapprobation; on peut penser que décocher a une interprétation inchoative ;

- La métaphore implique une idée d'hostilité : on trouve des adjectifs comme : hostile, haineux, courroucé, de désapprobation, acéré, inquisiteur ;

— Eil ne peut pas remplacer regard: *décocher un ceil sévère ;

- La réduction du verbe support est impossible, sinon on constate l'effacement de la métaphore : Le regard de Paul (à, sur) Jean vient de l'emploi en jeter ;

- Regard ne peut pas être thématisé : *Le regard de Paul se (darde, décoche) sur Jean.

Un emploi assez proche implique la métaphore d'une arme blanche : Paul plante son regard dans les yeux de Jean.

\subsection{Emploi : perception active : métaphore de l'attachement}

Paul a fixé (son, ses) regard(s) sur Jean

- L'objet désigne un humain, un concret ou un locatif ;

- La préposition est sur ;

- On trouve d'autres supports : attacher (préposition à) et visser (préposition à) ;

- Comme déterminants, on trouve le possessif singulier ou pluriel ; le défini est impossible, de même que un ou un-modif ;

- On observe des substantifs synonymes : fixer les yeux, visser les yeux mais pas de singulier? fixer son xil sur $N$, ?attacher l'œil à $N$; 
— L'effacement du verbe support implique la perte de la métaphore ;

- Une thématisation de regard est possible : Son regard s'est fixé sur Jean;

- Aspectuellement, il s'agit d'une interprétation résultative, durative et insistante : Son regard reste (fixé, attaché, vissé) sur Jean; Paul tient son regard fixé sur $N$; Paul a le regard fixé sur $N$.

\subsection{Emploi : perception active : métaphore du mouvement orienté}

Paul baisse son regard (sur Marie, vers le fond)

- L'objet désigne un humain, un concret ou un locatif ; le complément est obligatoire, sinon on a affaire à la suite plus ou moins figée : baisser les yeux au sens de ne pas vouloir regarder;

- Les prépositions peuvent être : sur, vers ;

- On trouve d'autres supports : lever, tourner, relever ;

- Déterminants : le possessif singulier et le défini le;

- Regard peut être remplacé par le pluriel yeux (mais pas au singulier);

- Le verbe support ne peut pas être réduit ;

- La prédicat peut être thématisé : Son regard se tourne vers, ?Son regard se baisse, Ses yeux se sont levés (baissés, tournés) vers $N$;

— L'aspect est ponctuel et duratif : Paul tient son regard (levé, baissé, *tourné).

Cet emploi a un présupposé : baisser son regard sur implique que l'on soit dans une position supérieure (le contraire pour lever les yeux sur). Peut-être ces constructions sont-elles des suites plus ou moins figées, où elles signifient approximativement avoir de l'intérêt pour, faire attention à ou demander la clémence de.

\subsection{Emploi : perception active : métaphore militaire (épée, pistolet)}

Paul a braqué son regard (sur le nouveau venu, vers la porte)

- En position de N1 on trouve un humain, un concret ou un locatif ;

- Autres supports possibles : diriger, pointer ;

- Les prépositions sont locatives et directionnelles : vers, en direction de, sur ;

- En position de déterminants, on trouve le possessif (singulier et pluriel), le défini ou encore un-modif ;

- Regard peut être remplacé par yeux (mais non par le singulier $\propto$ eil) ;

- Le verbe support peut être réduit, surtout si la préposition est nettement locative : Le regard de Paul en direction de; 
- Parmi les modifieurs, on trouve : réprobateur, de reproche, scrutateur, investigateur, *fixe, *fuyant;

- Une thématisation de regard est possible : Le regard de Paul se braque subitement vers $N$.

La métaphore est fondée, cette fois-ci, sur la notion d'arme à feu ou d'une épée : (braquer, pointer) (un pistolet, un revolver, une épée) (sur, vers) Nhum. Cet emploi implique une attitude agressive.

\subsection{Emploi : perception active : métaphore de causatif du mouvement}

\section{a) Déplacement}

Paul porte (son, ses regards) sur ce tableau

- L'objet désigne un humain, un concret, un locatif ou une situation ;

- D'autres supports sont possibles : promener, laisser traîner ;

- Les prépositions sont locatives : sur, vers, dans la direction de;

- Parmi les déterminants, on observe le possessif (singulier et pluriel), peut-être un-modif, le défini est impossible ;

- Regard a un synonyme : ses yeux (mais pas au singulier);

- Le verbe support ne peut pas être effacé ;

- Thématisation du prédicat nominal : Le regard de Paul se porte vers ce spectacle; Le regard de Paul se promène sur cette toile;

- L'aspect est duratif et progressif mais l'interprétation résultative est exclue : *Paul a le regard porté sur $N$; Paul, le regard porté sur $N$, s'avançait vers lui.

\section{b) Résultatif de déplacement}

Paul a posé son regard sur Marie

- L'objet désigne un humain, un concret ou un locatif ;

- La préposition est sur ;

- Parmi les déterminants, on trouve : le possessif(singulier et pluriel), le, un-modif;

- Prédicat synonyme : ses yeux (mais pas au singulier);

- Le regard de Paul sur Marie est-ce un effacement de poser ou jeter?;

- Thématisation du prédicat : Le regard de Paul s'est posé sur Marie;

- L'aspect est duratif et résultatif : Paul avait le regard posé sur $N$;

- Adjectifs appropriés : appuyé, attentif, perçant, profond. 


\section{c) Antidéplacement}

Paul arrête son regard sur $N$

- L'objet désigne un humain, un concret ou un locatif ;

- Autre support : peut-être fixer ;

- La préposition est sur ;

— Déterminants : possessif, *le, *un, *un-modif ;

- Synonymes : ses yeux (mais pas au singulier);

- Thématisation : (son regard, ses yeux) s'arrête(nt) sur ce tableau;

- Aspect : l'interprétation résultative n'est pas évidente ?Paul a (le regard, les yeux) arrêté(s) sur $N$.

\subsection{Emploi négatif : métaphore du détournement}

Paul détourne son regard de ce spectacle

- Objet : un humain, un concret ou un locatif ;

- Autre support possible : éloigner ;

- Préposition : de ;

- Déterminants : le possessif (singulier et pluriel), le défini le ;

- Synonymes du prédicat : yeux, ? oil ;

- Thématisation du prédicat : son regard se détourne de ce spectacle ;

- Adjectifs : effrayé, effaré, apeuré.

Il y a une lecture métaphorique : ne pas vouloir prendre en considération. La nature de détourner n'est pas claire : derrière le possessif il est difficile de trouver un support. Cf. Il a perdu sa bonne humeur = Il a perdu la bonne humeur qu'il avait. Dans $I l$ a détourné son regard de $N$, on ne peut pas postuler Il a détourné le regard qu'il avait jeté sur $N$.

\subsection{Restructurations}

Le substantif regard est en position de « complément » indirect. On a alors une restructuration du type :

Paul Vsup Dét regard Prép N1

Paul V N1 du regard 
Ces constructions sont souvent de nature aspectuelle. La nature du verbe est métaphorique et traduit :

a) un mouvement :

Paul accompagne la voiture du regard

Paul suit la voiture du regard

Paul parcourt le paysage du regard

b) un geste :

Paul embrasse le paysage du regard

c) un prédicat de « recherche »

Paul scrute le paysage du regard

Paul fouille le paysage du regard

d) autres métaphores

Paul couve Marie du regard

Paul dévore Marie du regard

Paul déshabille Marie du regard

\subsection{Autres thématisations}

Le regard de Paul est tombé sur une vielle photo

Son regard court le long de la crête

Son regard découvre un vaste paysage

Son regard rencontre celui de Marie

Son regard bute sur un détail inattendu

\subsection{Résumé conclusif}

1. Les différents emplois d'un prédicat peuvent relever de classes sémantiques très différentes : regard peut être un prédicat de perception, de propriété, de comportement ;

2. C'est très souvent le verbe support qui détermine la lecture du prédicat nominal ; ce verbe support dépend aussi des différentes métaphores utilisées ;

3. Chaque emploi prédicatif est défini par une détermination spécifique : il faudrait étudier les conditions dans lesquelles le possessif est seul possible ;

4. Il n'y d'effacement du verbe support que s'il n'y a pas perte d'information, en particulier aspectuelle ;

5. Il est difficile de prédire quand un prédicat nominal a un verbe associé ;

6. Les restructurations sont à étudier de façon systématique. 


\section{5. Étude systématique d'un prédicat verbal. Grille de description du verbe lire}

Les observations qui précèdent mettent en évidence que la syntaxe, la sémantique et le lexique ne sont pas des instances autonomes mais sont imbriqués de façon multiple. Les analyses qui suivent proposent une description globale et intégrée d'un emploi prédicatif donné.

\subsection{Un domaine d'arguments}

Un verbe ne peut être décrit qu'à l'aide des classes sémantiques de ses arguments (les classes d'objets) et non pas avec des classifieurs très généraux comme Nabstrait. Le verbe lire a deux emplois différents :

- lire/NO : hum/N1 : texte (article) ; support d'écriture (livre, journal)

- lire/NO : hum/N1 : missive (lettre)/N2 : à l'intention de destinataire/N3 : au sujet de, concernant Névé

Ces classes doivent évidemment être décrites en extension, à l'aide de listes qui permettent de générer toutes les phrases possibles avec ce prédicat :

- l'ensemble des textes : roman, poème, etc.

- l'ensemble des supports d'écriture : cahier, livre, journal, etc.

— l'ensemble des missives : message, lettre, dépêche, télégramme, etc.

\subsection{Un sens, d'où un ou des synonymes et une traduction}

Le schéma argumental ainsi défini, il est possible d'attribuer une signification au prédicat et, par conséquent, de lui adjoindre un synonyme et une traduction.

\subsection{Une forme morphologique du prédicat}

On note, dans cette rubrique, la forme morphologique que peut prendre un prédicat donné : verbe, nom, adjectif. Ici, le verbe a deux formes nominales associées : faire la lecture de et être lecteur de. Ces prédicats nominaux ont, du fait de leur statut morphologique, des propriétés spécifiques, il faut donc renvoyer à d'autres grilles qui les décrivent :

Paul a lu ce texte

Paul a effectué lecture de ce texte

Paul est le lecteur de ce texte 


\subsection{Une actualisation}

On consigne ici le numéro de la conjugaison du verbe et, dans les grilles associées décrivant les prédicats nominaux, la nature du verbe support. Lire correspond, dans la classification du DELAS/DELAF, à un verbe du type V94, qui décrit l'ensemble des formes que ce verbe peut prendre.

\subsection{Un système aspectuel}

Le verbe lire est un prédicat de « décodage » d'aspect duratif. On peut donc avoir des formes aspectuelles comme :

(commencer, continuer, poursuivre, finir, arrêter) de lire ce texte.

D'autres variantes peuvent être :

Il a mis (beaucoup, peu, assez de temps) à lire ce texte.

\subsection{Des transformations}

Le verbe lire a une syntaxe très régulière. On a donc toutes les transformations habituelles portant sur:

— le sujet : interrogatif : qui ?; pronom : il ; démonstratif : celui-ci, etc.

— l'objet : interrogatif : que? ; pronom : le, la ; démonstratif : celui-ci, celle-là, etc.

— la structure de la phrase : passif, interrogation en est-ce que?

\subsection{Un domaine}

Ici, langue générale.

\subsection{Un niveau de langue}

Lire a peu de synonymes du même niveau de langue. Déchiffrer implique un texte difficile à lire, feuilleter ou parcourir désignent une lecture rapide ou superficielle, bouquiner sélectionne en position argumentale un livre et est d'un niveau de langue différent. 


\section{9. Étude systématique des transformations portant sur une phrase}

Une grille, comme celle qui vient d'être décrite, peut subir un grand nombre de transformations qu'il faut décrire en extension, en vue d'une description complète des formes de phrases.

\subsubsection{Les transformations structurelles}

a) changements de catégories morphologiques des prédicats : nominalisation (lecture), adjectivation (lisible au passif) ;

b) interrogations portant sur le prédicat (est-ce que P), sur un argument (qui ? que ?) ou un circonstant (quand? pourquoi ? où ?) ;

c) négation portant sur le prédicat ne ... pas, ne ... plus et sur les arguments ne ... aucune, aucun ... ne, etc. ;

d) enchâssements, c'est-à-dire le phénomène de la récursivité, qui insère une phrase dans une position argumentale : Paul a lu que le président avait démissionné ;

e) pronominalisations portant sur les arguments nominaux ou phrastiques ( $\mathrm{il}$, le, les, leur, en, y) ;

f) relativation, transformation d'une phrase en un syntagme nominal : Le livre que j'ai lu est difficile.

\subsubsection{Les mises en évidence}

Elles peuvent concerner le prédicat, les arguments et, plus rarement, les déterminants. Leur rôle est de mettre l'accent sur un élément de la phrase en le plaçant en position initiale.

\section{- Mise en évidence du prédicat} extraposition du verbe : La neige tombe, Il tombe de la neige extraposition passive : Il est lu beaucoup de journaux ces derniers temps dislocation : Il a lu un journal, Ce qu'il a lu c'est un journal

\section{- Mise en évidence des arguments}

a) extraction : J'ai lu ce livre, C'est moi qui ai lu ce livre

b) détachement (dislocation) à gauche : Ce livre, je l'ai lu

c) détachement (dislocation) à droite : Je l'ai lu, ce livre

d) dislocation d'un argument phrastique : Ce que j'ai lu, c'est un livre 


\section{— Mise en évidence de déterminants}

J'ai lu des centaines de livres

J'ai lu des livres par centaines

\subsubsection{Les effacements}

Dans différentes conditions textuelles, un ou plusieurs des éléments du schéma prédicatif peuvent être omis, soit pour éviter une redite soit parce que l'information qu'il apporte est considérée comme non pertinente dans le contexte. On trouve ainsi :

a) l'effacement d'un argument : Pierre est en train de lire

b) l'effacement d'un prédicat: Pierre lit plus vite que moi

c) l'effacement de l'actualisation du prédicat : Pierre espère lire ce livre

\subsubsection{Les restructurations}

Une restructuration est un changement dans l'ordre canonique des arguments. Ces rotations correspondent à différents besoins d'expressivité. Le prédicat peut rester constant ou subir des modifications de forme.

\section{Restructurations sans modification de la forme du prédicat}

a) les restructurations contrastives : Jean lu un article et moi un livre

b) les permutations de longueur : J'ai lu à Paul le livre que je lui avais promis

\section{Restructurations avec modification de la forme du prédicat}

Passif :

Ce livre a été lu par l'ensemble des citoyens

Constructions à sujets réciproques :

Paul et Jean se lisent l'un l'autre

Tout emploi verbal devrait être examiné au regard de l'ensemble des paramètres qui ont été énumérés dans ce chapitre 5. On voit donc que la description complète d'un prédicat verbal, en vue du traitement automatique d'un emploi prédicatif donné, exige la recensement d'un très grand nombre de paramètres. Un des objectifs que l'on peut se proposer à la recherche linguistique, consiste à appliquer 
cette grille d'analyse à l'ensemble des prédicats. Des grilles appropriées aux prédicat nominaux et adjectivaux doivent être mises au point avec les mêmes précisions. C'est la seule condition d'une génération automatique possible.

\section{Description des connecteurs}

\subsection{Grille d'analyse}

Ce qui vient d'être dit s'applique essentiellement aux phrases simples, c'est-àdire celles qui ne mettent en jeu qu'un seul prédicat. Mais chacun sait qu'il existe des phrases complexes, impliquant plusieurs prédicats et qui nécessitent des descriptions spécifiques. C'est le cas quand il s'agit de connecteurs. Les paramètres d'analyse sont les suivants.

a) la suite est-elle figée ou non

Dans un cas on aura des locutions comme :

à telle enseigne que, au fur et à mesure que

et dans l'autre :

à cause de, dans le but de, avec le désir de

dont la forme générale est Prép Dét $N$ (de v, que P)

Quand le connecteur n'est pas figé, les paramètres d'analyse sont les suivants :

b) Y a-t-il une détermination ?

- anaphorique :

a) en vue de quoi, à la suite de quoi, en raison de cela, d'ici là

b) dans ce but, pour cette raison, à cet effet, à cette fin

— interrogative : dans quel but?, pour quelle raison ?, à quel moment?

— négative : à aucun moment, dans nulle autre but que de $V$

c) Y a-t-il un paradigme pour le substantif ?

à (le moment, l'instant, l'heure, la minute) où

pour (la raison, le motif) que $P$

de (façon, manière, sorte) que $P$

avec (la volonté, le dessein, l'intention) de $V$

d) Si le substantif est de nature prédicative, quel est son verbe support ? avoir l'intention de 
avoir le désir de

se fixer comme objectif de

être cause de

être la conséquence de

e) Ce substantif a-t-il un verbe ou un adjectif associé ?

avec le désir deV/ il a le désir de V/il désire V/il est désireux de V

à cause de N/être cause de N/causer $N$

avec la volonté de V/avoir le volonté de V/vouloir $V$

f) Y a-t-il d'autres thématisations ?

Jean a dit cela dans l'intention de convaincre

Convaincre était l'intention qu'avait Jean en disant cela

L'intention qu'avait Jean en disant cela était de convaincre

\subsection{Exemple de description le substantif but}

\subsubsection{Changement de la préposition}

dans le but de

avec le but de

\subsubsection{Détermination du substantif}

\subsubsection{Détermination affirmative}

\subsection{Détermination cataphorique}

Mc $=$ modifieur complétif (que $\mathbf{P}$, de $\mathbf{V W}$, de $\mathbf{N}$ )

$\mathrm{Ma}=$ modifieur adjectival

dans le but de réussir

dans le but avoué de réussir

dans un but de réussite

dans un but avoué de réussite

\subsection{Détermination anaphorique :}

dans ce but 
dans un tel but

dans un but pareil

dans un but de ce genre

sans un but similaire

dans le but qu'on vient de dire

dans un but identique

\subsubsection{Autres déterminations :}

\subsection{Négative :}

dans aucun but précis

dans nul autre but

\subsection{Interrogative :}

dans quel but?

\subsection{Exclamative :}

dans quel but!!

\subsection{Indéfinie :}

dans un certain but

6.2.3. Utilisation de verbes supports : avoir, avoir comme

Il a fait cela dans le but de réussir

Il a fait cela. Il avait le but de réussir

Il a fait cela. Il avait comme but de réussir

6.2.4. Autres verbes : se fixer, se donner, etc.

Il a fait cela. Il s'était fixé le but de réussir

Il a fait cela. Il s'était fixé comme le but de réussir

Il a fait cela. Il s'était donné le but de réussir

Il a fait cela. Il s'était donné comme but de réussir 


\subsubsection{Renversement de constructions}

Faire cela était son but

Il avait comme but de réussir en faisant cela

Faire cela constituait pour lui un but évident

But de cela : faire réfléchir

Le but de Luc est de convaincre

\subsubsection{Autres constructions}

Ce travail est à but lucratif

Ce travail a pour but de faire réfléchir

Ce but consiste à faire réfléchir

Dans le but suivant : faire réfléchir

Le but à long terme est éminemment politique

But de l'opération : renverser la dictature

But de la mancuvre : renverser la dictature

Comme on le voit, on aurait tort, de considérer les conjonctions comme des éléments isolés, des espèces particulières de locutions figées. Ici encore, on voit que les descriptions doivent être complètes et mettre en jeu l'ensemble des mécanismes qui sont en jeu dans une structure donnée.

\section{Les équivalences discursives}

\subsection{Le figement}

Tout le monde connait les travaux de Maurice Gross sur le figement. Son objectif était de noter toutes les constructions verbales qui n'avaient pas une syntaxe régulière, dont par exemple, le sujet ou l'un et l'autre des compléments constituaient des hapax. Voici un exemple mettant en jeu le verbe coucher :

coucher à la belle étoile

coucher en joue

coucher ensemble

coucher noir sur blanc

coucher par écrit

coucher sous les ponts 
coucher sur la dure

coucher sur le papier

coucher sur Poss testament

Ce travail de grande ampleur n'a pas pu être mené à bout, du fait de la mort de M. Gross. Il reste que ces listes regroupent des constructions qui n'ont pas de lien entre elles. Il s'agit simplement de dresser la liste des constructions qui n'ont pas de construction régulière et libre. Ces travaux étaient évidemment indispensables mais regroupent des suites qui ne constituent pas des ensembles homogènes. Il reste que ces travaux peuvent être poursuivis dans plusieurs directions. L'une d'elles est ce que j'appelle des équivalences discursives, c'est-à-dire des façons de s'exprimer identiques du point de vue de l'information et de la communication. Je vais donner une série d'exemple, en les classant en fonction de la catégorie grammaticale.

\title{
7.2. Constructions adjectivales
}

Certains adjectifs ont des constructions restreintes, en particulier du point de vue du complément en pour $N$, ce qui n'est évidemment pas prédictible :

\author{
c'est bien fait pour Nhum \\ c'est bien fait pour la gueule de Nhum \\ c'est bien fait pour la pomme de Nhum \\ c'est bien fait pour les fesses de Nhum \\ c'est bien fait pour les pieds de Nhum
}

Quand un objet n'est plus utilisable, on peut dire de lui :

il est bon pour la casse

il est bon pour la ferraille

il est bon pour la réforme

il est bon pour l'abattoir

il est bon pour le rebut

Parmi les constructions adjectivales, il faut faire un cas particulier des constructions intensives en comme $N$. L'intensité d'une propriété peut se traduire par un grand nombre de moyens linguistiques : le superlatif, des adverbes comme très, énormément, au plus haut point, etc. Avec les constructions en comme $N$, la comparaison intensive se fait avec un élément considéré comme prototypique de la qualité ou du défaut évoqué. 
blanc comme neige

blanc comme un cachet d'aspirine

blanc comme un linceul

blanc comme un linge

bête comme chou

bête comme Poss pieds

bete comme une cruche

bête comme une oie

con comme la lune

con comme un balai

con comme une baleine

con comme une bite

con comme une valise

\subsection{Constructions adverbiales}

Les constructions adverbiales sont très nombreuses du point de vue de leur sémantisme. Une énumération peut, par exemple, se terminer de la façon suivante, mettant en jeu l'adjectif dernier, terme d'une énumération :
en dernier lieu
en dernier recours
en dernier ressort
en dernière analyse
en dernière instance
en dernière minute
en dernière ressource

L'allure à laquelle on se déplace peut être rendue par le substantif pas, accompagné de certains adjectifs descriptifs :
à pas comptés
à pas de géant
à pas de loup
à pas de tortue
à pas feutrés
à pas mesurés 
L'activité ou l'énergie s'expliquent souvent par les situations particulières où l'on peut se trouver :

dans le feu de l'action
dans le feu de la bagarre
dans le feu de la discussion
dans le feu du combat

Certaines expressions sont des indications de temps ou d'aspect :

voici belle lurette

voici longtemps

voici un temps fou

voici une paille

voici une paye

Enfin, une expression comme et cetera, dans le cas d'une énumération, peut être remplacée par des suites du type :

et tout le bastringue

et tout le bataclan

et tout le bazar

et toute la compagnie

\subsection{Exclamations}

Le dégoût devant certaines situations pénibles peut se traduire par un assez grand nombre d'expressions :

quel bordel!

quel cirque!

quel foutoir!

quelle chiasse!

quelle horreur!

quelle merde!

L'étonnement, causé par une événement inattendu, s'exprime différemment :

tiens!

tiens donc!

tiens pardi! 
tiens tiens!

tenez!

\subsection{Verbes et adverbes}

Il y a là un domaine de rechercher d'une ampleur insoupçonnée :

blêmir de colère

blêmir d'épouvante

blêmir de honte

blêmir d'horreur

blêmir d'indignation

blêmir de peur

blêmir de rage

écumer de colère

écumer de fureur

écumer d'impatience

écumer d'indignation

Il en est de même de différentes façons dont on peut boire de l'alcool :

boire $N$ à grandes gorgées

boire $N$ à grandes lampées

boire $N$ à grands coups

boire $N$ à grands traits

boire $N$ à longs traits

boire $N$ à longues gorgées

boire $N$ à longues lampées

boire $N$ à petites gorgées

boire $N$ à petites lampées

boire $N$ à petits coups

boire $N$ à pleines carafes

boire $N$ à pleines cruches

boire $N$ à pleins tonneaux

boire $N$ à pleins verres

\subsection{Verbes avec compléments}

L'intensité peut se traduire par différents moyens lexicaux : 
coûter un argent dingue

coûter un argent fou

coûter un paquet

coûter un tas d'or

coûter une fortune

coûter une montagne d'or

coûter une somme dingue

coûter une somme folle

pleurer à chaudes larmes

pleurer comme un veau

pleurer comme une fontaine

pleurer comme une Madeleine

pleurer comme une vache

pleurer des larmes de sang

pleurer toutes les larmes de son corps

\title{
7.7. Verbes et adverbes
}

Cette combinaison est source d'équivalences considérables. Voici quelques exemples :

\author{
éclater en applaudissements \\ éclater en injures \\ éclater en larmes \\ éclater en pleurs \\ éclater en reproches \\ éclater en sanglots \\ travailler pour des clous \\ travailler pour des haricots \\ travailler pour des nèfles \\ travailler pour des prunes
}

\subsection{Verbes intensifs en comme}

bondir comme un cabri

bondir comme un chevreau

bondir comme un ressort

bondir comme un tigre 
saigner comme un bouf saigner comme un cochon saigner comme un porc

fumer comme un pompier fumer comme un sapeur fumer comme un troupier fumer comme une cheminée fumer comme une locomotive

\subsection{Verbes figés}

avoir une tête à claques

avoir une tête à gifles

avoir une tête d'enterrement

avoir une tête de cochon

avoir une tête de con

avoir une tête de croque-mort

avoir une tête de linotte

avoir une tête de mule

se fendre la gueule

se fendre la pêche

se fendre la poire

\subsection{Phrases figées}

Les phrases figées sont des constructions d'une particulière importance :

allez vous faire fiche!

allez vous faire foutre!

allez vous faire voir ailleurs!

ça biche

ça boume

ça colle

ça gaze

ça marche

ça roule

ça va 


\author{
ça va au poil \\ ça va barder \\ ça va barder pour ton matricule! \\ ça va chaudement \\ ça va chauffer \\ ça va chier \\ ça va péter \\ ça va péter le feu
}

\title{
Conclusion
}

Cet article avait pour objectif de faire le point sur les conditions d'une description linguistique susceptible de mener au traitement automatique des langues. Il $\mathrm{y}$ a à cela plusieurs conditions indispensables. Tout d'abord, il faut mettre au point un nombre total de critères permettant de décrire avec précision l'ensembles des éléments d'une phrases : prédicats, arguments, déterminants, modifieurs, adverbiaux. Il convient, ensuite, pour chaque classe de mettre au point la liste complète des éléments, sans oublier les suites figées. Il faut enfin, examiner les relations qui dépassent le cadre des phrases, comme on vient de le voir avec les équivalences discursives. Aucune description sérieuse et reproductible n'est possible en dehors d'une théorie.

\section{Références citées}

Anscombre, J.-Cl. (1995). Morphologie et représentation événementielle : le cas des noms de sentiment et d'attitude. Langue française, 105, 40-54.

Anscombre, J.-Cl. (2019). Figement, lexique et matrices lexicales. Cahiers de Lexicologie, $114,119-149$.

Bach, E. (1986). The algebra of events. Linguistics and Philosophy, 9, 5-16.

Baudet, S. (1990). Représentations d'états, d'événements et d'actions. Langages, 100, $45-64$.

Chomsky, N. (1965). Aspects of the Theory of Syntax. Cambridge, The Massachusetts Institute of Technology.

Delerm, Ph. (2018). Et vous avez eu beau temps? La perfidie ordinaire des petites phrases. Paris, Seuil. 
Desclés, J.-P. (1991). Archétypes cognitifs et types de procès. Travaux de Linguistique et de Philologie, 29, 171-195.

Dostie, G. (2019). Paramètres pour définir et classer les phrases préfabriquées. Cahiers de Lexicologie, 114, 27-63.

Dostie, G., \& Tutin, A. (2019). La phrase préfabriquée dans le paysage phraséologique. Cahiers de Lexicologie, 114, 11-25.

Dubois, J., \& Dubois-Charlier, Fr. (2004). Les locutions en français. Aix-en-Provence, inédit, chez les auteurs. https://www.modyco.fr/fr/base-documentaire/ressources/ jean-dubois/761-locutions-en-fran\%C3\%A7ais,-dubois-dubois-2004/file.html.

Franckel, J.-J. (1989). Étude de quelques marqueurs aspectuels du français. Genève, Droz.

Fuchs, C. (1991). Les typologies de procès : un carrefour théorique interdisciplinaire. Travaux de Linguistique et de Philologie, 29, 9-17.

Gaatone, D. (2004). Ces insupportables verbes supports ; le cas des verbes événementiels. Linguisticae Investigationes, 27(2), 239-253.

Giry-Schneider, J. (1987). Les prédicats nominaux en français : les phrases simples à verbes supports. Genève, Droz.

Gross, G. (1986). Les constructions nominales et l'élaboration d'un lexique-grammaire. Langue française, 69, 5-27 (en coll. avec R. Vivès).

Gross, G. (1989). Les constructions converses du français. Genève, Droz.

Gross, G. (1993). Trois applications de la notion de verbe support. L'information grammaticale, 59, 16-23.

Gross, G. (1995). À quoi sert la notion de partie de discours? In L. Basset \& M. Pérennee (Dir.), Les classes de mots. Traditions et perspectives (p. 217-231). Lyon, Presses universitaires de Lyon.

Gross, G. (1996a). Les expressions figées en français : des noms composés aux locutions. Paris, Ophrys.

Gross, G. (1996b). Prédicats nominaux et compatibilité aspectuelle. Langages, 121, $54-73$.

Gross, G. (2004). Pour un Bescherelle des prédicats nominaux. Linguisticae Investigationes, 27(2), 343-359.

Gross, G. (2009). Sémantique de la cause. Louvain-Paris, Peeters (en coll. avec R. Pauna et Fr. Valetopoulos).

Gross, G. (2012). Manuel d'analyse linguistique. Villeneuve-d'Ascq, Presses universitaires du Septentrion [Traduction espagnole : Manual de Analisis Lingüistico. Aproximation Sintactico-semantica al lexico (X. Blanco Escoda, Trad.). Editorial UOC, Barcelona, 2013].

Gross, G., \& Kiefer, F. (1995). La structure événementielle des substantifs. Folia Linguistica, 29(1-2), 43-65.

Gross, G., \& Prandi, M. (2004). La finalité : fondements conceptuels et genèse linguistique. «Champs languistiques ». Louvain-la-Neuve, Duculot [Traduction italienne : La finalità. Strutture concettuali e forme d'espressione in italiano (C. de Santis, Trad.). Firenze, Olschki, 2005].

Gross, M. (1975). Méthodes en syntaxe. Paris, Hermann.

Gross, M. (1981). Les bases empiriques de la notion de prédicat sémantique. Langages, 63, $7-52$. 
Guenthner, Fr. (1998). Constructions, classes et domaines : concepts de base pour un dictionnaire de l'allemand. Langages, 131, 45-55.

Harris, Z. S. (1976). Notes du cours de syntaxe. Paris, Seuil.

Kaufer, M. (2019). Les actes de langage stéréotypés. Essai de synthèse critique. Cahiers de Lexicologie, 114, 149-173.

Kiefer, F. (1974). Essais de sémantique générale. Paris, Mame.

Kleiber, G. (1990). La sémantique du prototype. Paris, Presses universitaires de France.

Lees, R. B. (1960). The grammar of English Nominalizations. La Haye, Mouton.

Le Pesant, D. (2019). Suggestions méthodologiques et outils de traitement de corpus pour l'étude des phrases préfabriquées des intercations. Cahiers de Lexicologie, 114, 93-119.

Le Pesant, D., \& Mathieu-Colas, M. (1998). Introduction aux classes d'objets. Langages, $131,6-63$.

Mel'cuk, I. (2004). Verbes supports sans peine. Linguisticae Investigationes, 27(2), $203-219$.

Prandi, M. (1998). Contraintes conceptuelles sur la distribution. Langages, 131, 34-44.

Tutin, A. (2019). Phrases préfabriquées des intercations : quelques observations sur le corpus CLAPI. Cahiers de Lexicologie, 114, 63-93.

Vendler, Z. (1968). Adjectives and Nominalizations. La Haye, Mouton.

Vivès, R. (1983). Avoir, prendre, perdre : constructions à verbes supports et extensions aspectuelles. Thèse de $3^{\mathrm{e}}$ cycle, Université Paris VIII et LADL.

Von Polenz, P. (1963). Funktionsverben im heutigen Deutsch. Wirkendes Wort, 5 (Beihefte zur Zeitschrift). Düsseldorf, Pädagogischer Verlag Schwann. 